\title{
Three-dimensional assessment of nasal changes after maxillary advancement with impaction using stereophotogrammetry
}

\author{
Gokhan Coban ${ }^{\mathrm{a}}$ (1) \\ Ibrahim Yavuz \\ Busra Karadas ${ }^{a}$ \\ Ahmet Emin Demirbas ${ }^{b}$
}

${ }^{a}$ Department of Orthodontics, Faculty of Dentistry, Erciyes University, Kayseri, Turkey

${ }^{\mathrm{b}}$ Department of Oral and Maxillofacial Surgery, Faculty of Dentistry, Erciyes University, Kayseri, Turkey
Objective: To evaluate the changes in the nose in three dimensions after le Fort 1 osteotomy in patients with skeletal Class 111 malocclusion. Methods: The subjects were 40 adult patients (20 females and 20 males; mean age, $20.3 \pm 3.0$ years; range, 17.0 to 31.1 years) who underwent one-piece Le Fort 1 osteotomy with maxillary advancement and impaction treatment for maxillary hypoplasia. The mean maxillary advancement was $4.56 \pm 1.34 \mathrm{~mm}$, and the mean maxillary impaction was $2.03 \pm 1.04 \mathrm{~mm}$. Stereophotogrammetry was used to acquire three-dimensional images before and at least 6 months after surgery. Results: Alare (Al) and alare curvature (Ac) points had moved vertically and anterolaterally postoperatively. A significant increase was observed in the nasal ala width and alar base width, and no changes were noted in the columellar length, nasolabial angle, and nasal area. There was a significant relationship between maxillary impaction and nasal ala width and horizontal and sagittal positions of the bilateral $\mathrm{Al}$ and Ac. The only relationship found was between maxillary advancement and postoperative sagittal location of the subnasale and pronasale. Conclusions: Nasal soft tissues were highly affected by the vertical movement of the maxilla; however, the soft tissue responses were individual-dependent.

[Korean J Orthod 2020;50(4):249-257]

Key words: 3D stereophotogrammetry, Class 111 orthognathic surgery, Soft tissue nasal changes

Received December 30, 2019; Revised April 14, 2020; Accepted April 14, 2020.

Corresponding author: Gokhan Coban.

Lecturer, Department of Orthodontics, Faculty of Dentistry, Erciyes University, B Block Floor 3, Melikgazi, Kayseri 38039, Turkey.

Tel +090 3522076600 (29103) e-mail dtgokhancoban@hotmail.com

How to cite this article: Coban G, Yavuz I, Karadas B, Demirbas AE. Three-dimensional assessment of nasal changes after maxillary advancement with impaction using stereophotogrammetry. Korean J Orthod 2020;50:249-257.

(C) 2020 The Korean Association of Orthodontists.

This is an Open Access article distributed under the terms of the Creative Commons Attribution Non-Commercial License (http://creativecommons.org/licenses/by-nc/4.0) which permits unrestricted non-commercial use, distribution, and reproduction in any medium, provided the original work is properly cited. 


\section{INTRODUCTION}

The major goals of orthognathic surgery treatment are as follows: formation of facial esthetics and functional occlusion, improvement and/or maintenance of airway volume and periodontal health, healthy temporomandibular joints, and elimination of the patient's major complaint. ${ }^{1}$ For determination of facial aesthetics, the nose is a very important structure as the center of the face $^{2}$ and all surgical interventions on the midface affect the nose. Undesirable changes in the appearance of the nose were the most important cause of patient dissatisfaction in maxillary orthognathic surgery. ${ }^{3}$ Therefore, careful preoperative examinations of the nose and implementation of a treatment plan based on these findings will yield effective results.

Previous articles have used two-dimensional (2D) methods, such as radiographs and photographs, to show the aesthetic proportion of the profile and front of the face. ${ }^{4}$ However, since the human body is three-dimensional (3D), face depth and shape cannot be accurately evaluated using 2D measures. Recent developments in technology have produced a variety of 3D techniques, such as 3D computed tomography, ${ }^{5}$ laser scanning, ${ }^{6}$ 3D facial morphometry, ${ }^{7}$ and stereophotogrammetry, ${ }^{5,8}$ to capture facial topography and overcome the disadvantages of traditional photographic and radiographic methods. Stereophotogrammetry is a fast, accurate, highly reproducible, and non-invasive 3D digital camera system that can easily yield not only linear but also topographic, angular, and areal measurements in 3D.,8

The traditional belief is that maxillary advancement often leads to shortening of the nasal ridge and an increase in the nasolabial angle due to nasal tip elevation, ${ }^{9}$ and maxillary impaction with ${ }^{10}$ or without maxillary advancement ${ }^{11}$ often leads to widening of the nose. This may cause an unpleasant appearance in patients with a wide nose preoperatively.

Many studies have been conducted with 3D digital imaging systems to examine changes in the nose after orthognathic surgery in relation to maxillary surgery and the nasal tip, nasolabial angle, alar base width, and coordinate pronasale changes. ${ }^{5,8,12,13}$ However, these studies failed to investigate 3D nose symmetry in detail after orthognathic surgery treatment. Therefore, the aims of this study were as follows: (1) perform a comprehensive evaluation of the 3D changes of the nose using stereophotogrammetry after maxillary advancement with impaction in patients with skeletal Class 111 malocclusion; (2) assess the magnitude of 3D symmetry and postoperative changes of the alare and alare curvature points according to the reference planes; and (3) explore the correlation between the hard and the soft tissue changes after orthognathic surgery treatment.

\section{MATERIALS AND METHODS}

\section{Subjects}

A retrospective study was designed and implemented to address the research objectives. The study protocol was approved by the Erciyes University Clinical Research Ethics Committee (Approval No: 2018/83). The alar base width for the first ten patients was evaluated in a pilot study, and a power analysis ( $G$ *Power Software ver. 3.1.9.2; Universität Düsseldorf, Düsseldorf, Germany) was performed. A sample size of 39 patients had 90\% power to detect significant differences at a 0.05 significance level. The study population consisted of all patients presenting for evaluation and management of skeletal Class 111 malocclusion and aged between 17 years 0 months and 31 years 1 month.

The subjects were 40 adult Turkish patients (20 females and 20 males; mean age, $20.3 \pm 3.0$ years) who underwent orthodontic treatment and maxillary surgery for skeletal Class 111 malocclusion at Department of Orthodontics and Oral Maxillofacial Surgery, Faculty of Dentistry, Erciyes University, Kayseri, Turkey. All patients underwent maxillary orthognathic surgery, including one-piece Le Fort type 1 maxillary advancement and maxillary total impaction, to achieve a Class 1 dentoskeletal relationship. The mean maxillary advancement was $4.56 \pm 1.34 \mathrm{~mm}$ and the mean maxillary impaction was $2.03 \pm 1.04 \mathrm{~mm}$. To measure the amount of surgical movement on the cephalometric film, a horizontal reference plane (HRP) was formed $7^{\circ}$ from the sella-nasion plane, and the vertical reference plane (VRP) passing through the sella was drawn perpendicular to this horizontal plane (Figure 1). ${ }^{6}$ The perpendicular distances of the hard tissue A point to VRP and the posterior nasal spina (PNS) to HRP between before and after the orthognathic surgery revealed the amount of movement. The hard tissue A point was used for evaluation of advancement of the maxilla and the PNS point was used to evaluate the impaction. The occlusal plane angle was measured to check impaction and was $15.36^{\circ} \pm 7.31^{\circ}$ pre-surgery and $14.97^{\circ} \pm 5.31^{\circ}$ post-surgery.

None of the patients had severe facial asymmetry, history of trauma, congenital anomalies, and/or other craniofacial disorders. Patients who underwent an anterior nasal spina (ANS) reduction, an alar base cinch suture, or a rhinoplasty surgery were excluded. All patients had a body mass index between 18 and $26 \mathrm{~kg} / \mathrm{m}^{2}$. The 3D images of the patients were acquired with stereophotogrammetry (3dMD Face; 3dMD, Atlanta, GA, USA) 1 day before and at least 6 months $(8.91 \pm 2.81$ months $)$ after surgery on the same day that the cephalometric films were taken, in the natural head position (NHP), centric occlusion, and with the lips at rest. For acquisition of NHP, ${ }^{14}$ the patients were firstly recommended 
to walk around and relax. Next, the patients performed gradually decreasing forward and backward head oscillations until they settled their heads into a self-balanced position. They were then asked to look at the reflection of their eyes in a mirror. Soft tissue nasal changes were evaluated using the $3 \mathrm{dMDvultus}$ (3dMD) software, and the file format was ".tsb."

For the initial registration, the 3D images of the patients before and after surgery were aligned manually, before using the automatic registration feature of the software for fine-tuning the alignment. After the 3D images were adequately positioned manually, the most optimal surface areas of the face that would not be

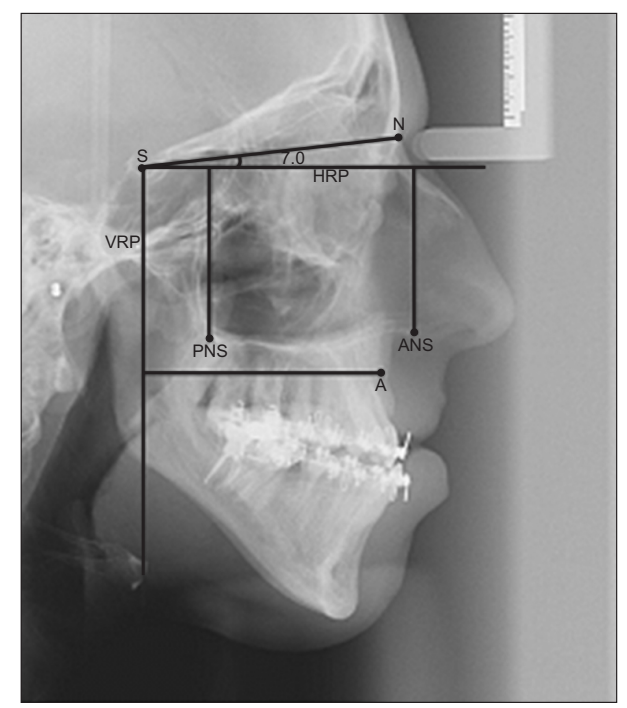

Figure 1. Evaluation of surgical quantities of hard tissues. N, Nasion; S, sella; A, A point; ANS, anterior nasal spina; PNS, posterior nasal spina; HRP, horizontal reference plane; VRP, vertical reference plane.
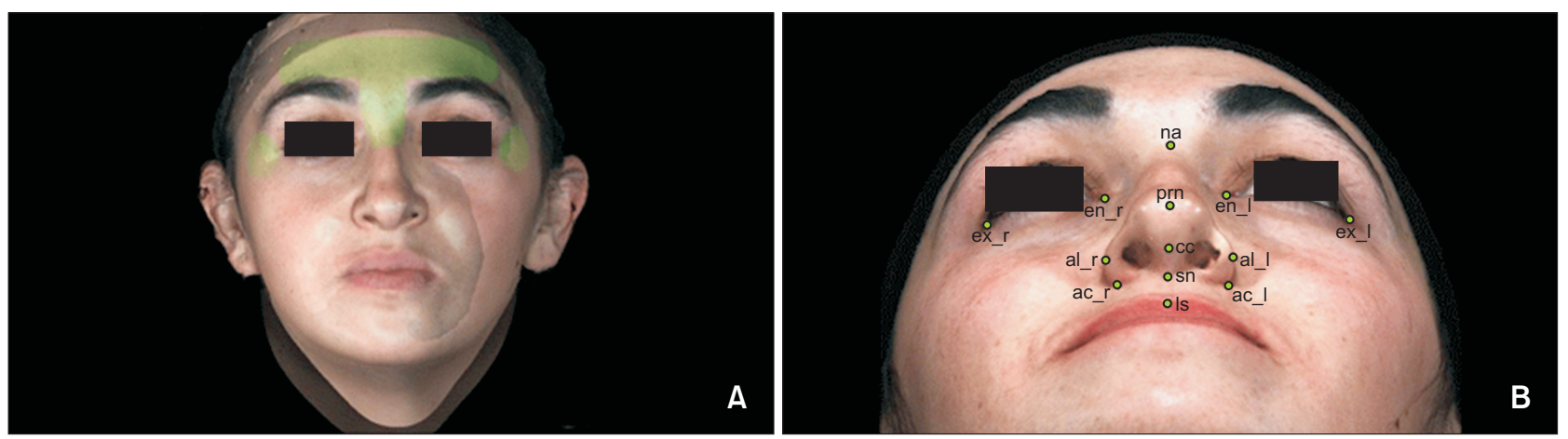

Figure 2. A, Reference markers used for superimposition: forehead area (the wide surface over the eyebrows), nasion area (the corresponding soft tissue point from the nasal root to the nasal hump), and exocanthion (the $3 / 4$ lateral regions of the exocanthion). B, Soft tissue landmarks. na, Soft tissue nasion; en, endocanthion; ex, exocanthion; prn, pronasale; cc, columella constructed point; al, alare; ac, alare curvature; sn, subnasale; ls, labiale superior; r, right; l, left. influenced by surgery were selected: the widest surface on the forehead, the surface from the nasal root to the nasal hump, and the lateral regions of the exocanthion (Figure 2A). ${ }^{15}$ The 3D image superimpositions were shown to be reliable with an average root mean square (RMS) error of 0.24 and a range of 0.12 to 0.45 (smaller than 0.5$)$. These data were clinically acceptable and consistent with previously used methods. ${ }^{16}$

The landmarks for the soft tissues (Figure 2B) and reference planes (Figure $3 \mathrm{~A}$ ) are defined ${ }^{15}$ in Table 1 . The $3 \mathrm{D}$ coordinates of the facial soft tissue landmarks were manually placed using $3 \mathrm{dMDvultus}$ ( $3 \mathrm{dMD}$ ) software. The following facial soft tissue landmarks were considered:

- Median points: nasion, na; pronasale, prn; subnasale, sn; columella constructed, cc; labiale superior, ls.

- Lateral points: (right and left sides indicated by subscripts_r and _l, respectively) endocanthion, en_r and en_l; exocanthion, ex_r and ex_l; alare, al_r and al_l; alar curvature, ac_r and ac_l.

In determining the 3D coordinate system settings, ${ }^{17}$ left and right pupil points are connected by a segment that defines the $x$ (left-right)-axis. The $Y Z$ plane perpendicularly bisects the $\mathrm{x}$-axis. The $\mathrm{z}$-axis is the anteroposterior axis of the face on the YZ plane. The $y$ (vertical)axis segment passes perpendicularly through the center of the $\mathrm{Z}$-axis on the $\mathrm{YZ}$ plane. The imaginary center point of $3 \mathrm{D}$ facial geometry is the zero point $(\mathrm{y}=0, \mathrm{z}=$ 0 , and $y z=0$ ). The $x z$ plane is perpendicular to the $y z$ plane and passes through the zero point. The xy plane passes through the zero point and is perpendicular to the $y z$ and $x z$ planes (Figure $3 A$ ). The 3D coordinate values $(x, y$, and $z$ ) of the landmarks were acquired using the coordinate system of the imaginary center of facial geometry (the zero point).

The changes in the $3 \mathrm{D}$ coordinates and directions of 


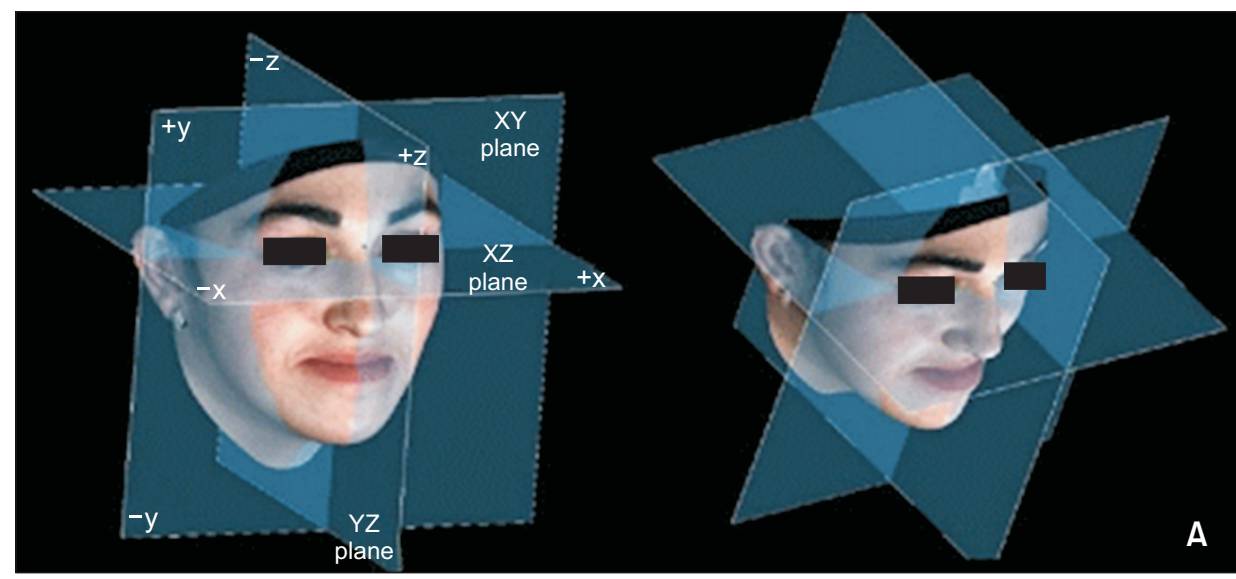

Figure 3. A, Axial reference plane passing through both endocanthions when the patients had their natural head positions. Sagittal reference plane passing through na, perpendicular to the axial and coronal reference planes; coronal reference plane pass-

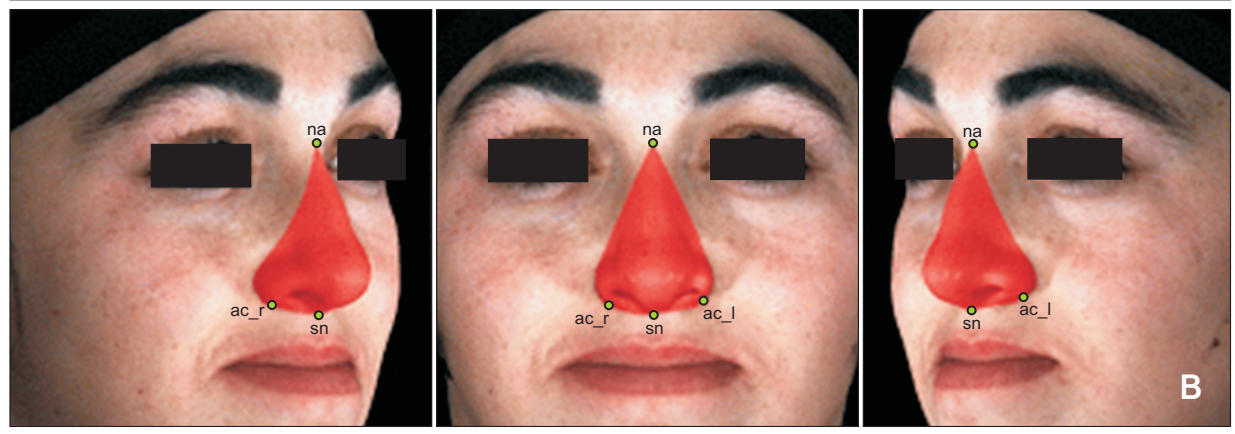
ing through both exocanthions, perpendicular to the axial and sagittal planes. $\mathbf{B}$, External nasal surface area measurement was computed following soft tissue nasal borders.

na, Soft tissue nasion; ac, alare curvature; sn, subnasale; r, right; I, left.

Table 1. Definitions of soft tissue landmarks and reference planes

\begin{tabular}{|c|c|c|}
\hline Soft tissue landmark & Abbreviation & Definition \\
\hline Nasion & na & The point in the midline of both the nasal root and the frontonasal suture \\
\hline Endocanthion & en & The soft tissue point located at the inner commissure of the each eye fissure \\
\hline Exocanthion & ex & The soft tissue point located at the outer commissure of the each eye fissure \\
\hline Pronasale & prn & The most prominent point on the nasal tip \\
\hline Columella constructed point & $\mathrm{cc}$ & The midpoint of the columella crest at the level of the nostril top points \\
\hline Alare & al & The most lateral point on each alar contour \\
\hline Alare curvature & ac & The point located at the facial insertion of each alar base \\
\hline Subnasale & sn & $\begin{array}{l}\text { The midpoint on the nasolabial soft tissue contour between the columella crest } \\
\text { and the upper lip }\end{array}$ \\
\hline Labiale superior & ls & The midpoint of the vermilion line of the upper lip \\
\hline \multicolumn{3}{|l|}{ Reference plane } \\
\hline Axial plane & & $\begin{array}{l}\text { A plane constructed with the right endocanthion and the left endocanthion on } \\
\text { the natural head position, parallel to the floor }\end{array}$ \\
\hline Coronal plane & & $\begin{array}{l}\text { A plane constructed with the right exocanthion and the left exocanthion, } \\
\text { perpendicular to the axial plane }\end{array}$ \\
\hline Sagittal plane & & A plane constructed with nasion, perpendicular to the axial and coronal planes \\
\hline
\end{tabular}

landmarks are shown in Table 2. The preoperative differences in each right and left al and ac points according to the reference planes were compared to the postoperative differences (Table 3). Table 3 shows the changes in bilateral $\mathrm{Al}$ and $\mathrm{Ac}$ between reference planes and pa- tients after orthognathic surgery treatment.

The external nasal surface area was the computed sum of the surfaces between na, the following soft tissue border of the right alar-facial sulcus, the groove and right ac, sn, and left ac, the following soft tissue border 
Coban et al • 3D assesment of nasal soft tissue changes

Table 2. Changes in three-dimensional coordinates of landmarks

\begin{tabular}{|c|c|c|c|c|c|c|}
\hline \multirow{2}{*}{ Variable } & \multicolumn{2}{|c|}{$\mathbf{X}$} & \multicolumn{2}{|c|}{$\mathbf{Y}$} & \multicolumn{2}{|c|}{$\mathbf{Z}$} \\
\hline & $\Delta \mathrm{T}(\mathrm{T} 1-\mathrm{T} 0)$ & $p$-value & $\Delta T(T 1-T 0)$ & $p$-value & $\Delta \mathrm{T}(\mathrm{T} 1-\mathrm{T} 0)$ & $p$-value \\
\hline Prn & $-0.08 \pm 0.95$ & 0.928 & $1.14 \pm 1.63$ & $0.000^{* * *}$ & $1.35 \pm 0.87$ & $0.000^{* * *}$ \\
\hline $\mathrm{Sn}$ & $-0.14 \pm 0.75$ & 0.459 & $0.60 \pm 0.99$ & $0.000^{* * *}$ & $2.24 \pm 1.59$ & $0.000 * * *$ \\
\hline Al_r & $-1.22 \pm 0.90$ & $0.000^{* * *}$ & $1.21 \pm 1.20$ & $0.000^{* * *}$ & $2.64 \pm 1.55$ & $0.000^{* * *}$ \\
\hline Al_l & $1.25 \pm 1.28$ & $0.000^{* * *}$ & $1.07 \pm 1.72$ & $0.002^{* *}$ & $2.82 \pm 1.29$ & $0.000^{* * *}$ \\
\hline Ac_r & $-1.42 \pm 0.90$ & $0.000^{* * *}$ & $0.78 \pm 1.10$ & $0.000^{* * *}$ & $3.67 \pm 1.43$ & $0.000^{* * *}$ \\
\hline Ac_l & $1.54 \pm 1.27$ & $0.000^{* * *}$ & $1.12 \pm 1.33$ & $0.000^{* * *}$ & $3.32 \pm 1.73$ & $0.000^{* * *}$ \\
\hline
\end{tabular}

Values are presented as mean \pm standard deviation.

Paired-samples $t$-test were performed.

T0, Presurgery; $\mathrm{T} 1$, postsurgery; $\Delta \mathrm{T}$, amount of change after surgery (T1-T0); $\mathrm{x}$-axis, left (no sign), right (minus sign); $\mathrm{y}$-axis, superior (no sign), inferior (minus sign); z-axis, anterior (no sign), posterior (minus sign).

${ }^{* *} p<0.01,{ }^{* * *} p<0.001$.

See Table 1 for definitions of the other landmarks.

Table 3. Three-dimensional nasal symmetry measurements according to the reference planes

\begin{tabular}{cccc}
\hline Variable & T0 & T1 & $\boldsymbol{p}$-value \\
\hline Sagittal (x) (mm) & & & \\
Al_l/Al_r & $-0.78 \pm 1.77$ & $-0.80 \pm 2.18$ & 0.892 \\
Ac_l/Ac_r & $-1.06 \pm 2.06$ & $-1.18 \pm 2.21$ & 0.574 \\
Axial (y) (mm) & & & \\
Al_l/Al_r & $0.64 \pm 1.40$ & $0.50 \pm 0.86$ & 0.487 \\
Ac_l/Ac_r & $0.39 \pm 0.93$ & $0.74 \pm 0.93$ & $0.004^{* *}$ \\
Coronal (z) (mm) & & & \\
Al_l/Al_r & $0.56 \pm 1.48$ & $0.38 \pm 1.73$ & 0.339 \\
Ac_l/Ac_r & $0.53 \pm 1.32$ & $0.88 \pm 1.30$ & 0.339 \\
\hline
\end{tabular}

Values are presented as mean \pm standard deviation.

Paired $t$-test was performed.

T0, Pre-treatment; T1, post-treatment; Al_l/r, alare left/right; Ac_l/r, alare curvature left/right; Point_l/Point_r, difference between the distances from point_l and point_r to the reference plane.

${ }^{* *} p<0.01$.

of the left alar-facial groove, the sulcus and na (Figure 3B). The changes in the 6 coordinate values, 6 difference values, and the 5 linear, 1 angular, and 1 areal measurements (Tables 2, 3 and 4) were calculated, and the correlations between hard and soft tissues were evaluated, (Table 5) in this study.

\section{Statistical analysis}

All the data in this study were normally distributed according to the Shapiro-Wilk test. The samples were evaluated as a single group and analyzed by the pairedsamples t-test, since there was no statistically significant difference in the soft tissue changes between male and female patients in the independent-samples $t$-test. A $p$-value $<0.05$ was considered statistically significant. The correlations between the soft-tissue and hardtissue movements in the horizontal, vertical, and sagittal planes were analyzed by Pearson correlation analysis. The measurements of ten patient's digital images were performed twice by the same examiner (GC) one month apart. Intraclass correlation coefficients for nasal measurements were between 0.85 and 0.98 , indicating a strong positive correlation and sufficient reliability. ${ }^{18}$

\section{RESULTS}

The 3D coordinate ( $\mathrm{x}, \mathrm{y}$, and $\mathrm{z}$ ) values of the landmarks were obtained by using a coordinate system. In the horizontal (x) plane, bilateral al and ac showed significant lateral movement while there were no significant changes in prn and sn. In the vertical (y) plane, all soft tissue landmarks moved upward, and in the sagittal (z) plane, all soft tissue landmarks moved forward significantly (Table 2). The average increase of $0.4^{\circ}$ in the occlusal plane angle was not statistically significant. Table 3 shows the difference between pre- and postoperative measurements to the reference planes of the right and left al and ac in 3 dimensions. Only the vertical (y) preoperative difference between the right and left ac increased significantly after the operation (Table 3). There was a significant increase in the 3D linear measurements of the nose alar width and alar base width, and there were no significant changes in the nasal bridge length, columellar length, nasolabial angle, nasal tip protrusion, and external nasal surface area (Table 4). Table 5 shows the correlation between hard tissue and soft tissue changes. There was a significant relationship between maxillary impaction and nasal alar width, and lateral and anterior movements of the bilateral al and 
Table 4. Changes in nasal measurements

\begin{tabular}{lccl}
\hline \multicolumn{1}{c}{ Variable } & T0 & T1 & p-value \\
\hline Nose/alar width (Al_r-Al_l) $(\mathrm{mm})$ & $34.70 \pm 3.77$ & $37.04 \pm 3.90$ & $0.000^{* * *}$ \\
Alar base width (Ac_r-Ac_l) $(\mathrm{mm})$ & $29.05 \pm 3.11$ & $32.09 \pm 3.56$ & $0.000^{* * *}$ \\
Nasal bridge length (Na-Prn) $(\mathrm{mm})$ & $46.94 \pm 5.46$ & $46.78 \pm 5.46$ & 0.500 \\
Nasal tip protrusion $(\mathrm{Sn}-\mathrm{Prn})(\mathrm{mm})$ & $19.97 \pm 4.87$ & $19.64 \pm 4.64$ & 0.130 \\
Columellar length $(\mathrm{Sn}-\mathrm{Cc})(\mathrm{mm})$ & $9.96 \pm 2.58$ & $9.90 \pm 2.57$ & 0.872 \\
Nasolabial angle $(\mathrm{Ls}-\mathrm{Sn}-\mathrm{Cc})\left({ }^{\circ}\right)$ & $103.41 \pm 15.32$ & $103.02 \pm 11.95$ & 0.766 \\
Nose area $\left(\mathrm{cm}^{2}\right)$ & $24.81 \pm 4.01$ & $24.65 \pm 4.32$ & 0.712 \\
\hline
\end{tabular}

Values are presented as mean \pm standard deviation.

Paired $t$-test was performed.

T0, Pre-treatment; T1, post-treatment; Al_l/r, alare left/right; Ac_l/r, alare curvature left/right; Na, soft tissue nasion; Prn, pronasale; Sn, subnasale; Cc, columella constructed; Ls, labiale superior.

*** $p<0.001$.

Table 5. Correlations of soft-tissue and hard-tissue changes

\begin{tabular}{|c|c|c|c|c|}
\hline \multirow[t]{2}{*}{ Variable } & \multicolumn{2}{|c|}{$\begin{array}{l}\text { Maxillary advancement } \\
\text { (mean } 4.56 \pm 1.34 \mathrm{~mm})\end{array}$} & \multicolumn{2}{|c|}{$\begin{array}{c}\text { Maxillary impaction } \\
\text { (mean } 2.03 \pm 1.04 \mathrm{~mm})\end{array}$} \\
\hline & Movement (\%) & $p$-value & Movement (\%) & $p$-value \\
\hline Prn_y & 24 & 0.133 & 13 & 0.410 \\
\hline Prn_z & 31 & $0.029 *$ & 14 & 0.768 \\
\hline Sn_y & 21 & 0.189 & 14 & 0.525 \\
\hline $\mathrm{Sn} \_\mathrm{z}$ & 33 & $0.034^{*}$ & 1 & 0.318 \\
\hline Al_x & 10 & 0.896 & 35 & $0.026^{*}$ \\
\hline Al_y & 12 & 0.588 & 12 & 0.486 \\
\hline Al_z & 25 & 0.145 & 43 & $0.003^{* *}$ \\
\hline Ac_x & 25 & 0.434 & 33 & $0.048^{*}$ \\
\hline Ac_y & 22 & 0.692 & 5 & 0.803 \\
\hline Ac_z & 25 & 0.131 & 40 & $0.003^{* *}$ \\
\hline Nasal alar width & 10 & 0.542 & 36 & $0.038^{*}$ \\
\hline Alar base width & 20 & 0.075 & 32 & 0.063 \\
\hline
\end{tabular}

Mean values for bilateral points (Al and $\mathrm{Ac}$ ) are presented.

Pearson correlation analysis was performed.

Prn, Pronasale; Sn, subnasale; Al, alare; Ac, alare curvature; _x, transversal direction; _y, vertical direction; _z, anteroposterior direction.

${ }^{*} p<0.05,{ }^{* *} p<0.01$.

ac. The only significant positive correlation was noted between maxillary advancement and anterior movement of sn and prn.

\section{DISCUSSION}

The aim of this study was to evaluate the effect of maxillary advancement and impaction on 3D nasal symmetry and changes in the soft tissues of the nose. We used 3D stereophotogrammetry, a fast and reliable technique $e^{5}$ that is accurate enough for clinical use with a clinical error of 0.2 to $1.0 \mathrm{~mm}^{8}$ compared to $0.5-1.0$ $\mathrm{mm}$ for laser scanning. ${ }^{6}$

Imaging is a commonly used method in orthodontics and maxillofacial surgery to record, evaluate, and display the size and shape of craniofacial structures. The 3D imaging systems include 3D computed tomography, ${ }^{5}$ laser scanning, ${ }^{6}$ 3D facial morphometry, ${ }^{7}$ and stereophotogrammetry. ${ }^{5,8}$ The advantages of stereophotogrammetry include fast capture times, which decrease the likelihood that 3D images will be affected by body movements; minimal cooperation required from patients; no expo- 
sure to radiation; accurate and precise measurements obtained by rotating the images in the desired direction; high reproducibility; the provision for linear, topographic, areal, and volumetric measurements; and the facility to perform registration on pre- and post-surgery images. ${ }^{19}$ Disadvantages are the need for additional imaging methods (such as cone-beam computed tomography and cephalometric roentgens) for evaluation of hard tissues, ${ }^{20}$ high cost, the requirement of a large specialized area to set up the machinery, the difficulties of displaying complex areas and anatomical gaps, the need to calibrate the system frequently, and the impossibility of acquiring dynamic images. ${ }^{8}$ The 3D image collection and evaluations were performed at least 6 months after surgery to allow complete resolution of edema and establishment of soft-tissue adaptation to maxillary osteotomy. ${ }^{21}$ In assessments of 3D coordinates, Baik and $\mathrm{Kim}^{6}$ observed a mean 1.3-mm movement in al_r to the right side and in al_l to the left side, and both of these moved anteriorly by a mean distance of $2.3 \mathrm{~mm}$, which was similar to our results for al and ac.

Consistent with previous reports, a tendency toward an upward and forward movement of the prn was observed in this study. ${ }^{8,9,12,20}$ Schendel and Williamson ${ }^{9}$ reported a mean nasal tip elevation of $2.4 \mathrm{~mm}$ and an impaction of $6.4 \mathrm{~mm}$ in patients who underwent mean maxillary advancement. In this study, the mean 1-mm upward movement of prn may have been due to the differences in surgical quantities.

The differences in the distances of the right and left al and ac points relative to the reference planes before and after treatment and horizontal changes of prn and sn were examined to evaluate 3D nasal symmetry changes. These values were considered smaller post-operatively with no deviations observed at prn and sn. Only the difference between the vertical $(\mathrm{y})$ positions of the right and left ac points increased by $0.3 \mathrm{~mm}$ after the operation; this may be due to ac points that were closer to the osteotomy line and the soft tissues underlying these points being thin. The increases were statistically significant; however, we observed that it was clinically insignificant.

ANS is an important component of nasal tip protrusion, ${ }^{21}$ and when ANS is left untouched, as in our study, larger quantities of nasal tip movement could be seen. ${ }^{22}$ However, Gassmann et al. ${ }^{23}$ claimed that there was no significant relationship between the presence or absence of ANS and nasal tip protrusion.

An increase was reported in the nasal alar width and alar base width after maxillary advancement surgery by both McCance et al., ${ }^{24}$ who used a 3D laser scanner, and Ferrario et al., ${ }^{7}$ who used 3D facial morphometry. Baik and $\mathrm{Kim}^{6}$ reported a mean alar widening of $2 \mathrm{~mm}$, and Chung et al. ${ }^{12}$ reported a significant increase in alar width and alar base width even in cases where a cinch suture was properly used. Interalar width was always greater post-surgery, probably because the nasal base, like sn, was pushed forward by maxillary advancement surgery. ${ }^{7}$ Honrado et al. ${ }^{8}$ reported equivocal results, both decreases and increases, with nasal tip protrusion and columellar length, and the nasolabial angle was the only measurement showing a significant increase in patients who underwent maxillary advancement and impaction. Chung et al. ${ }^{12}$ reported a mean decrease of $1 \mathrm{~mm}$ in nasal tip protrusion, which was significant. This study showed an insignificant decrease in nasal tip protrusion, which could have occurred because sn was closer to the underlying hard tissue than to prn.

Misir et al. ${ }^{22}$ reported that there was no significant change in the nasolabial angle in 27 patients who were treated with bimaxillary surgery. Radney and $\mathrm{Jacobs}^{25}$ indicated that the change in the nasolabial angle was associated with the amount and direction of the maxillary surgery. No clockwise or counterclockwise rotation was made in our patients, and no significant change was found in the nasolabial angle.

The nasal bridge length that was measured from the na to the prn showed a tendency to decrease post-surgery, which was possibly due to changes in the direction of the prn. ${ }^{10,20}$ There was no significant change in the nose area after orthognathic surgery, which was similar to the report by Sforza et al. ${ }^{26}$

The ratios of the soft-tissue to hard-tissue changes in the prn and sn were 31\% and 33\% sagittally in our study. The respective measurements in the study by Baik and $\mathrm{Kim}^{6}$ were $30 \%$ and $57 \%$ and $81 \%$ and $29 \%$ in the study by Soncul and Bamber. ${ }^{27}$ In this study, the mean amounts of maxillary advancement and impaction were 4.6 and $2.0 \mathrm{~mm}$, respectively, while they were 1.0 to $3.0 \mathrm{~mm}$ in the study by Baik and $\mathrm{Kim}^{6}$ and $3 \mathrm{~mm}$ in the study by Soncul and Bamber. ${ }^{27}$ The differences in the ratios were most probably caused by the degrees of surgical movement. The reason for comparing the facial midline soft tissues following maxillary movements to the lateral soft tissues was that the semicircular form of the maxilla and the muscular attachments were more tightly connected to the bone in the midline. ${ }^{27}$ Freihofer $^{28} \mathrm{ob}-$ served that the sn and prn advanced by 50\% and 25\% with the maxilla, respectively.

Sarver and Weissman ${ }^{21}$ reported that the soft tissue changes associated with maxillary impaction were minimal. However, we found a significant vertical decrease in all nasal landmarks (prn, sn, al, and ac) measured. The ratios of the transversal al and ac changes relative to the maxillary impaction were 35\% and 33\%; the sagittal change ratios were $43 \%$ and $40 \%$, respectively. There was a significant correlation between nasal alar width and maxillary impaction. Previous studies suggested that 
the probability of erroneous measurements in the vertical direction was greater than those in other directions, and the correlations with the vertical changes were difficult to predict. ${ }^{29,30}$ Soft tissue response differences might be due to the amounts of hard tissue movements, muscle structures, racial, and biological differences in the subjects, or unique variations in the soft tissue responses.

The limitations of the present study were the small sample size in each group and the lack of a control group. To make predictions of nasal changes according to maxillary skeletal changes, a larger sample size and control group of untreated patients would be needed in future studies.

The results of this study suggest that clinicians can predict the effect of orthognathic surgical interventions for the maxilla on the nasal structures and 3D nasal symmetry via a preliminary assessment and use this to guide surgical planning to improve patient outcomes. However, despite the increased use and advancement of 3D imaging systems, soft tissue predictions, especially in the nose, are still quite limited, ${ }^{8}$ and it is not always possible to anticipate the nasal changes precisely after maxillary surgery. ${ }^{2}$

\section{CONCLUSION}

Patients should be thoroughly informed about nasal changes after orthognathic surgery and the possibility of additional nasal surgical procedures before surgery. 3D stereophotogrammetry may be a valuable and useful tool in evaluating the nose in 3 dimensions.

The following conclusions can be reached from this study:

- Maxillary impaction was found to be correlated with changes in the horizontal and anteroposterior location of bilateral alare and alare curvature and nasal ala width measurements, while maxillary advancement was correlated only with changes in the anteroposterior location of the pronasale and subnasale.

- Alare and alare curvature points moved laterally, and an increase was observed in nose width.

- Nasal symmetry did not change after surgery, with the exception of the left and right vertical position of the alare curvature.

- Both pronasale and subnasale moved upwards; however, pronasale moved to a greater extent.

- The external nasal surface area did not change.

\section{CONFLICTS OF INTEREST}

No potential conflict of interest relevant to this article was reported.

\section{REFERENCES}

1. Arnett GW, McLaughlin RP. Facial and dental planning for orthodontists and oral surgeons. London: Mosby; 2004. p.4.

2. Schendel SA, Carlotti AE Jr. Nasal considerations in orthognathic surgery. Am J Orthod Dentofacial Orthop 1991;100:197-208.

3. Naini FB, Gill DS. Orthognathic surgery: principles, planning and practice. Chichester: John Wiley \&t Sons; 2017. p. 417.

4. Larrabee WF Jr. Facial analysis for rhinoplasty. Otolaryngol Clin North Am 1987;20:653-74.

5. van Loon B, van Heerbeek N, Bierenbroodspot F, Verhamme L, Xi T, de Koning MJ, et al. Threedimensional changes in nose and upper lip volume after orthognathic surgery. Int J Oral Maxillofac Surg 2015;44:83-9.

6. Baik HS, Kim SY. Facial soft-tissue changes in skeletal Class 111 orthognathic surgery patients analyzed with 3-dimensional laser scanning. Am J Orthod Dentofacial Orthop 2010;138:167-78.

7. Ferrario VF, Sforza C, Schmitz JH, Santoro F. Threedimensional facial morphometric assessment of soft tissue changes after orthognathic surgery. Oral Surg Oral Med Oral Pathol Oral Radiol Endod 1999;88:549-56.

8. Honrado CP, Lee S, Bloomquist DS, Larrabee WF Jr. Quantitative assessment of nasal changes after maxillomandibular surgery using a 3-dimensional digital imaging system. Arch Facial Plast Surg 2006;8:2635.

9. Schendel SA, Williamson LW. Muscle reorientation following superior repositioning of the maxilla. J Oral Maxillofac Surg 1983;41:235-40.

10. Mitchell C, Oeltjen J, Panthaki Z, Thaller SR. Nasolabial aesthetics. J Craniofac Surg 2007;18:756-65.

11. Guenthner TA, Sather AH, Kern EB. The effect of Le Fort 1 maxillary impaction on nasal airway resistance. Am J Orthod 1984;85:308-15.

12. Chung C, Lee Y, Park KH, Park SH, Park YC, Kim KH. Nasal changes after surgical correction of skeletal Class 111 malocclusion in Koreans. Angle Orthod 2008;78:427-32.

13. Choi JW, Lee JY, Oh TS, Kwon SM, Yang SJ, Koh KS. Frontal soft tissue analysis using a 3 dimensional camera following two-jaw rotational orthognathic surgery in skeletal class 111 patients. J Craniomaxillofac Surg 2014;42:220-6.

14. Solow B, Tallgren A. Natural head position in standing subjects. Acta Odontol Scand 1971;29:591-607.

15. Lim YK, Chu EH, Lee DY, Yang IH, Baek SH. Threedimensional evaluation of soft tissue change gradients after mandibular setback surgery in skeletal 
Class 111 malocclusion. Angle Orthod 2010;80:896903.

16. Lin HH, Chiang WC, Lo LJ, Sheng-Pin Hsu S, Wang $\mathrm{CH}$, Wan SY. Artifact-resistant superimposition of digital dental models and cone-beam computed tomography images. J Oral Maxillofac Surg 2013; 71:1933-47.

17. Kim YK, Lee NK, Moon SW, Jang MJ, Kim HS, Yun PY. Evaluation of soft tissue changes around the lips after bracket debonding using three-dimensional stereophotogrammetry. Angle Orthod 2015;85:83340.

18. Liu ZY, Yu J, Dai FF, Jiang RP, Xu TM. Threedimensional changes in lip vermilion morphology of adult female patients after extraction and nonextraction orthodontic treatment. Korean J Orthod 2019;49:222-34.

19. Rasse M, Forkert G, Waldhäusl P. Stereophotogrammetry of facial soft tissue. Int J Oral Maxillofac Surg 1991;20:163-6.

20. Betts NJ, Vig KW, Vig P, Spalding P, Fonseca RJ. Changes in the nasal and labial soft tissues after surgical repositioning of the maxilla. Int J Adult Orthodon Orthognath Surg 1993;8:7-23.

21. Sarver DM, Weissman SM. Long-term soft tissue response to LeFort 1 maxillary superior repositioning. Angle Orthod 1991;61:267-76.

22. Misir AF, Manisali M, Egrioglu E, Naini FB. Retrospective analysis of nasal soft tissue profile changes with maxillary surgery. J Oral Maxillofac Surg 2011;69:e190-4.

23. Gassmann CJ, Nishioka GJ, Van Sickels JE, Thrash
WJ. A lateral cephalometric analysis of nasal morphology following Le Fort 1 osteotomy applying photometric analysis techniques. J Oral Maxillofac Surg 1989;47:926-30.

24. McCance AM, Moss JP, Fright WR, James DR, Linney AD. A three dimensional analysis of soft and hard tissue changes following bimaxillary orthognathic surgery in skeletal 111 patients. Br J Oral Maxillofac Surg 1992;30:305-12.

25. Radney LJ, Jacobs JD. Soft-tissue changes associated with surgical total maxillary intrusion. Am J Orthod 1981;80:191-212.

26. Sforza C, Peretta R, Grandi G, Ferronato G, Ferrario VF. Soft tissue facial volumes and shape in skeletal Class 111 patients before and after orthognathic surgery treatment. J Plast Reconstr Aesthet Surg 2007;60:130-8.

27. Soncul M, Bamber MA. Evaluation of facial soft tissue changes with optical surface scan after surgical correction of Class 111 deformities. J Oral Maxillofac Surg 2004;62:1331-40.

28. Freihofer HP Jr. Changes in nasal profile after maxillary advancement in cleft and non-cleft patients. J Maxillofac Surg 1977;5:20-7.

29. Mansour S, Burstone C, Legan H. An evaluation of soft-tissue changes resulting from Le Fort 1 maxillary surgery. Am J Orthod 1983;84:37-47.

30. Koh CH, Chew MT. Predictability of soft tissue profile changes following bimaxillary surgery in skeletal class 111 Chinese patients. J Oral Maxillofac Surg 2004;62:1505-9. 